\title{
No Labeling Quantum Mechanics of Indiscernible Particles
}

\author{
G. Domenech ${ }^{1 *}$ F. Holik ${ }^{1}$, L. Kniznik ${ }^{1}$ and D. $\mathrm{Krause}^{2 \dagger}$ \\ September 11, 2018 \\ ${ }^{1}$ Instituto de Astronomía y Física del Espacio (IAFE) \\ Casilla de Correo 67, Sucursal 28, 1428 Buenos Aires, Argentina \\ ${ }^{2}$ Departamento de Filosofia - Universidade Federal de Santa Catarina, 88040-900 \\ Florianópolis-SC, Brazil.
}

\begin{abstract}
Our aim in this paper is to show an example of the formalism we have developed to avoid the label-tensor-product-vector-space-formalism of quantum mechanics when dealing with indistinguishable quanta. States in this new vector space, that we call the Q-space, refer only to occupation numbers and permutation operators act as the identity operator on them, reflecting in the formalism the unobservability of permutations, a goal of quasi-set theory.
\end{abstract}

Key words: quasi-sets, Fock-space, quantum indistinguishability.

\section{Introduction}

In quantum mechanics (QM), the state space of a system of indistinguishable quanta is constructed by means of the product of the individual spaces. The usual procedure is to first 'label' the state space of each quanta, then making the tensor product and, finally, imposing a symmetrization postulate by hand. This procedure has been largely criticized in the literature (see [5] for a detailed study). To go further in this discussion, we have explicitly constructed (based on the quasi-set theory $\mathfrak{Q}$-see below) a formalism within

${ }^{*}$ Fellow of the Consejo Nacional de Investigaciones Científicas y Técnicas (CONICET)

${ }^{\dagger}$ Fellow of the Conselho Nacional de Desenvolvimento Científico e Tecnológico (CNPq) 
which we can obtain the same results as in the standard formalism of quantum mechanics without appealing to artificial labelings [2], [3], [5]. In this paper we develop a paradigmatic application - namely, the evaluation of the correlations between the spin components in a two particles state - to exemplify its use. The paper is organized as follows. We first briefly review, in Section 2, quasi-set theory and its motivations. In Section 3 we outline the construction of the state space in the new formalism. In Section 4 we develop the example, and Section 5 contains the conclusions.

\section{The basic ideas of quasi-set theory}

We briefly review here the main ideas of quasi-set theory $\mathfrak{Q}$ following mainly [7]. Intuitively speaking, $\mathfrak{Q}$ is obtained by applying ZFU-like (ZermeloFraenkel plus Urelemente) axioms to a basic domain composed of $m$-atoms (the new ingredients that stand for indistinguishable quanta, and to which the usual concept of identity does not apply), $M$-atoms and aggregates of them. The theory still admits a primitive concept of quasi-cardinal, which intuitively stands for the 'quantity' of objects in a collection. This is made so that certain quasi-sets $x$ (in particular, those whose elements are q-objects) may have a quasi-cardinal, written $q c(x)$, but not an associated ordinal. It is also possible to define a translation from the language of ZFU into the language of $\mathfrak{Q}$ in such a way so that there is a 'copy' of ZFU in $\mathfrak{Q}$ (the 'classical' part of $\mathfrak{Q}$ ). In this copy, all the usual mathematical concepts can be defined (inclusive the concept of ordinal for the $\mathfrak{Q}$-sets, the copy of standard sets in $\mathfrak{Q}$ ), and the $\mathfrak{Q}$-sets turn out to be those quasi-sets whose transitive closure (this concept is like the usual one) does not contain $\mathrm{m}$ atoms.

In $\mathfrak{Q}$, 'pure' quasi-sets have only $m$-atoms as elements (although these elements may be not always indistinguishable from one another), and to them it is assumed that the usual notion of identity cannot be applied (the expressions $x=y$ and its negation, $x \neq y$, are not well formed formulas if either $x$ or $y$ stand for $m$-atoms). Notwithstanding, there is a primitive relation $\equiv$ of indistinguishability having the properties of an equivalence relation, and a defined concept of extensional identity, not holding among $m$ atoms, which has the properties of standard identity of classical set theories. More precisely, we write $x={ }_{E} y$ ( $x$ and $y$ are extensionally identical) iff they are both qsets having the same elements (that is, $\forall z(z \in x \leftrightarrow z \in y)$ ) or they are both $M$-atoms and belong to the same qsets (that is, $\forall z(x \in z \leftrightarrow y \in z)$ ). From now on, we shall use the symbol "=" for the extensional equality, 
except when explicitly mentioned.

Since the elements of a quasi-set may have properties (and satisfy certain formulas), they can be regarded as indistinguishable without turning to be identical (that is, being the same object), that is, $x \equiv y$ does not entail $x=y$. Since the relation of equality (and the concept of identity) does not apply to $m$-atoms, they can also be thought of as entities devoid of individuality. For details about $\mathfrak{Q}$ and about its historical motivations, see [5, Chap. 7].

One of the main features of $\mathfrak{Q}$ is its ability to take into account in 'set-theoretical terms' the non observability of permutations in quantum physics, which is one of the most basic facts regarding indistinguishable quanta. In standard set theories, if $w \in x$, then of course $(x-\{w\}) \cup$ $\{z\}=x$ iff $z=w$. That is, we can 'exchange' (without modifying the original arrangement) two elements iff they are the same element, by force of the axiom of extensionality. But in $\mathfrak{Q}$ there is a theorem guarantying the unobservability of permutations; in other words,

Theorem 2.1 Let $x$ be a finite quasi-set such that $x$ does not contain all indistinguishable from $z$, where $z$ is an m-atom such that $z \in x$. If $w \equiv z$ and $w \notin x$, then there exists $w^{\prime}$ such that $\left(x-z^{\prime}\right) \cup w^{\prime} \equiv x$

Here $z^{\prime}$ and $w^{\prime}$ stand for a quasi-set with quasi-cardinal 1 whose only element is indistinguishable (but not identical) from $z$ and $w$ respectively.

\section{The state space $\mathbb{V}_{Q}$}

In QM, the mathematical interpretation of a physical system is a complex separable Hilbert space $\mathcal{H}$. Quantum systems of distinguishable many particles are mathematically represented by the tensor products of their individual Hilbert spaces [1], the so called 'labeled-tensor-product' of state spaces. To pick up physical states from the whole set allowed by the labeled product, a symmetrization postulate is added that restricts the states available for the particles by imposing that, if particles are indistinguishable, then they can only access symmetrized (with respect to particle interchange) states if they are bosons, or antisymmetrized states if they are fermions. This trick of first labeling and then restricting the available states allows to reproduce quantum statistics satisfactorily, without dropping particle indexation. But this procedure may be criticized, in particular for the necessity of first labeling the state spaces of the indistinguishable quanta and later masking this labeling with the addition of a postulate. Sometimes it is argued that the 
usual procedure to construct the set of possible states should be replaced by another one that does not index particles, for example the Fock-space formalism. However the formal construction of the Fock-space does use the standard set theoretical framework, which presupposes classical individuality on its foundations. This seems to be, thus, not a genuine solution [6]. To solve it, we have proposed an alternative procedure ( $[2],[3])$ that resembles that of the Fock-space formalism but based on $\mathfrak{Q}$, thus genuinely avoiding artificial labeling.

Let us recall that the usual procedure in the Fock-space formalism is to define the fundamental (vacuum) state $|0\rangle$ as the eigenvector of the particle number operator $N_{k}=a_{k}^{\dagger} a_{k}$ with 0 eigenvalue, being $a_{k}^{\dagger}$ and $a_{k}$ the creation and annihilation operators of particles of $k$-type respectively, which satisfy commutation relations for bosons and anticommutation relations for fermions. The complete basis for $\mathcal{H}$ is obtained by successive application of $a^{\dagger}$ to the vacuum state. All operators and wave functions may be written in terms of $a_{k}^{\dagger}$ and $a_{k}$ [8].

We outline now the construction of the state space $\mathbb{V}_{Q}$ that respects indistinguishability in all steps, mainly following [2]. We shall be working within $\mathfrak{Q}$. Let us consider a quasi-set $\epsilon=\left\{\epsilon_{i}\right\}_{i \in I}$, where $I$ is an arbitrary collection of indexes (this makes sense in the 'classical part' of $\mathfrak{Q}$ ). We take the elements $\epsilon_{i}$ to represent the eigenvalues of a physical magnitude of interest. Consider then the quasi-functions $f$ (this concept generalizes that of function), $f: \epsilon \longrightarrow \mathcal{F}_{p}$, where $\mathcal{F}_{p}$ is the quasi-set formed of finite and pure quasi-sets. $f$ is the quasi-set formed of ordered pairs $\left\langle\epsilon_{i} ; x\right\rangle$ with $\epsilon_{i} \in \epsilon$ and $x \in \mathcal{F}_{p}$. Let us choice these quasi-functions in such a way that whenever $\left\langle\epsilon_{i_{k}} ; x\right\rangle$ and $\left\langle\epsilon_{i_{k^{\prime}}} ; y\right\rangle$ belong to $f$ and $k \neq k^{\prime}$, then $x \cap y=\emptyset$. Let us further assume that the sum of the quasi-cardinals of the quasi-sets which appear in the image of each of these quasi-functions is finite, and then, $q c(x)=0$ for every $x$ in the image of $f$, except for a finite number of elements of $\epsilon$. Let us call $\mathcal{F}$ the quasi-set formed of these quasi-functions. If $\left\langle x ; \epsilon_{i}\right\rangle$ is a pair of $f \in \mathcal{F}$, we will interpret that the energy level $\epsilon_{i}$ has occupation number $q c(x)$. These quasi-functions will be represented by symbols such as $f_{\epsilon_{i_{1}} \epsilon_{i_{2}} \ldots \epsilon_{i m}}$ (or by the same symbol with permuted indexes). This indicates that the levels $\epsilon_{i_{1}} \epsilon_{i_{2}} \ldots \epsilon_{i_{m}}$ are occupied. It will be taken as convention that if the symbol $\epsilon_{i_{k}}$ appears $j$-times, then the level $\epsilon_{i_{k}}$ has occupation number $j$. The levels that do not appear have occupation number zero.

It is important to point out that the order of the indexes in $f_{\epsilon_{i_{1}} \epsilon_{i_{2}} \ldots \epsilon_{i_{n}}}$ has no meaning at all because up to now, there is no need to define any particular order in $\epsilon$, the domain of the quasi-functions of $\mathcal{F}$. Nevertheless, 
we may define an order in the following way. For each quasi-function $f \in \mathcal{F}$, let $\left\{\epsilon_{i_{1}} \epsilon_{i_{2}} \ldots \epsilon_{i_{m}}\right\}$ be the quasi-set formed by the elements of $\epsilon$ such that $\left\langle\epsilon_{i_{k}}, X\right\rangle \in f$ and $q c(X) \neq 0(k=1 \ldots m)$. We call $\operatorname{supp}(f)$ this quasi-set (the support of $f$ ). Then consider the pair $\langle o, f\rangle$, where $o$ is a bijective quasifunction $o:\left\{\epsilon_{i_{1}} \epsilon_{i_{2}} \ldots \epsilon_{i_{m}}\right\} \longrightarrow\{1,2, \ldots, m\}$. Each of these quasi-functions $o$ define an order on $\operatorname{supp}(f)$. For each $f \in \mathcal{F}$, if $q c(\operatorname{supp}(f))=m$, then, there are $m$ ! orderings. Then, let $\mathcal{O} \mathcal{F}$ be the quasi-set formed by all the pairs $\langle o, f\rangle$, where $f \in \mathcal{F}$ and $o$ is a a particular ordering in $\operatorname{supp}(f)$. Thus, $\mathcal{O} \mathcal{F}$ is the quasi-set formed by all the quasi-functions of $\mathcal{F}$ with ordered support. For this reason, $f_{\epsilon_{i_{1}} \epsilon_{i_{2}} \ldots \epsilon_{i_{n}}} \in \mathcal{O} \mathcal{F}$ refers to a quasifunction $f \in \mathcal{F}$ and a special ordering of $\left\{\epsilon_{i_{1}} \epsilon_{i_{2}} \ldots \epsilon_{i_{n}}\right\}$. For the sake of simplicity, we will use the same notation as before. But now the order of the indexes is meaningful. It is also important to remark, that the order on the indexes must not be understood as a labeling of particles, for it easy to check, as above, that the permutation of particles does not give place to a new element of $\mathcal{O F}$. This is so because a permutation of particles operating on a pair $\langle o, f\rangle \in \mathcal{O F}$ will not change $f$, and so, will not alter the ordering. We will use the elements of $\mathcal{O F}$ later, when we deal with fermions.

A linear space structure is required to adequately represent quantum states. To equip $\mathcal{F}$ and $\mathcal{O} \mathcal{F}$ with such a structure, we need to define two operations " $\star$ " and "+", a product by scalars and an addition of their elements, respectively. Call $C$ the collection of quasi-functions which assign to every $f \in \mathcal{F}$ (or $f \in \mathcal{O} \mathcal{F}$ ) a complex number (again, built in the 'classical part' of $\mathfrak{Q}$ ). That is, a quasi-function $c \in C$ is a collection of ordered pairs $\langle f ; \lambda\rangle$, where $f \in \mathcal{F}$ (or $f \in \mathcal{O F}$ ) and $\lambda$ a complex number. Let $C_{0}$ be the subset of $C$ such that, if $c \in C_{0}$, then $c(f)=0$ for almost every $f \in \mathcal{O F}$ (i.e., $c(f)=0$ for every $f \in \mathcal{O} \mathcal{F}$ except for a finite number of quasi-functions). We can define in $C_{0}$ a sum and a product by scalars in the same way as it is usually done with functions as follows:

Definition 3.1 Let $\alpha, \beta$ and $\gamma \in \mathcal{C}$, and $c, c_{1}$ and $c_{2}$ be quasi-functions of $C_{0}$, then

$$
(\gamma * c)(f):=\gamma(c(f)) \text { and }\left(c_{1}+c_{2}\right)(f):=c_{1}(f)+c_{2}(f)
$$

The quasi-function $c_{0} \in C_{0}$ such that $c_{0}(f)=0$, for any $f \in F$, acts as the null element of the sum, for $\left(c_{0}+c\right)(f)=c_{0}(f)+c(f)=0+c(f)=c(f), \forall f$. With the sum and the multiplication by scalars defined above we have that $\left(C_{0},+, *\right)$ is a complex vector space. Each one of the quasi-functions of $C_{0}$ should be interpreted in the following way: if $c \in C_{0}$ (and $c \neq c_{0}$ ), let $f_{1}, f_{2}, f_{3}, \ldots, f_{n}$ be the only functions of $C_{0}$ which satisfy $c\left(f_{i}\right) \neq 0(i=$ 
$1, \ldots, n)$. These quasi-functions exist because, as we have said above, the quasi-functions of $C_{0}$ are zero except for a finite number of quasi-functions of $\mathcal{F}$. If $\lambda_{i}$ are complex numbers which satisfy that $c\left(f_{i}\right)=\lambda_{i}(i=1, \ldots, n)$, we will make the association $c \approx\left(\lambda_{1} f_{1}+\lambda_{2} f_{2}+\cdots+\lambda_{n} f_{n}\right)$. The symbol $\approx$ must be understood in the sense that we use this notation to represent the quasi-function $c$. The idea is that the quasi-function $c$ represents the pure state which is a linear combination of the states represented by the quasi-functions $f_{i}$ according to the interpretation given above.

In order to calculate probabilities and mean values, we have to introduce a scalar product, in fact two of them: $\circ$ for bosons and $\bullet$ for fermions, thus obtaining two (normed) vector spaces $\left(\mathbb{V}_{Q}, \circ\right)$ and $\left(\mathbb{V}_{Q}, \bullet\right)$ :

Definition 3.2 Let $\delta_{i j}$ be the Kronecker symbol and $f_{\epsilon_{i_{1}} \epsilon_{i_{2}} \ldots \epsilon_{i_{n}}}$ and $f_{\epsilon_{i_{1}^{\prime}} \epsilon_{i_{2}^{\prime}} \ldots \epsilon_{i_{m}^{\prime}}}$ two basis vectors, then

$$
f_{\epsilon_{i_{1}} \epsilon_{i_{2}} \ldots \epsilon_{i_{n}}} \circ f_{\epsilon_{i_{1}^{\prime}} \epsilon_{i_{2}^{\prime}}^{\prime} \ldots \epsilon_{i_{m}^{\prime}}}:=\delta_{n m} \sum_{p} \delta_{i_{1} p i_{1}^{\prime}} \delta_{i_{2} p i_{2}^{\prime}} \ldots \delta_{i_{n} p i_{n}^{\prime}}
$$

The sum is extended over all the permutations of the set $i^{\prime}=\left(i_{1}^{\prime}, i_{2}^{\prime}, \ldots, i_{n}^{\prime}\right)$ and for each permutation $p, p i^{\prime}=\left(p i_{1}^{\prime}, p i_{2}^{\prime}, \ldots, p i_{n}^{\prime}\right)$.

This product can be easily extended over linear combinations.

Definition 3.3 Let $\delta_{i j}$ be the Kronecker symbol, $f_{\epsilon_{i_{1}} \epsilon_{i_{2}} \ldots \epsilon_{i_{n}}}$ and $f_{\epsilon_{i_{1}^{\prime}} \epsilon_{i_{2}^{\prime}} \ldots \epsilon_{i_{m}^{\prime}}}$ two basis vectors, then

$$
f_{\epsilon_{i_{1}} \epsilon_{i_{2}} \ldots \epsilon_{i_{n}}} \bullet f_{\epsilon_{i_{1}^{\prime}} \epsilon_{i_{2}^{\prime}} \ldots \epsilon_{i_{m}^{\prime}}}:=\delta_{n m} \sum_{p} \sigma_{p} \delta_{i_{1} p i_{1}^{\prime}} \delta_{i_{2} p i_{2}^{\prime}} \ldots \delta_{i_{n} p i_{n}^{\prime}}
$$

where: $s^{p}=+1$ if $p$ is even and $s^{p}=-1$ if $p$ is odd.

The result of this second product $\bullet$ is an antisymmetric sum of the indexes which appear in the quasi-functions. In order that the product is well defined, the quasi-functions must belong to $\mathcal{O F}$. Once this product is defined over the basis functions, we can extend it to linear combinations, in a similar way as for bosons. If the occupation number of a product is more or equal than two, then the vector has null norm. As it is a vector of null norm, the product of this vector with any other vector of the space would yield zero, and thus the probability of observing a system in a state like it vanishes. This means that we can add to any physical state an arbitrary linear combination of null norm vectors for they do not contribute to the scalar product, which is the meaningful quantity. 
With these tools and using the language of $\mathfrak{Q}$, the formalism of $\mathrm{QM}$ may be completely rewritten giving a straightforward answer to the problem of giving a formulation of QM in which intrinsical indistinguishability is taken into account from the beginning, without artificially introducing extra postulates. We make the following association in order to turn the notation similar to that of the standard formalism. For each quasifunction $f_{\epsilon_{i_{1}} \epsilon_{i_{2}} \ldots \epsilon_{i_{n}}}$ of the quasi-sets $\mathcal{F}$ or $\mathcal{O F}$ constructed above, we will write $\left.\alpha f_{\epsilon_{i_{1}} \epsilon_{i_{2}} \ldots \epsilon_{i_{n}}}:=\alpha \mid \epsilon_{i_{1}} \epsilon_{i_{2}} \ldots \epsilon_{i_{n}}\right)$ with the obvious corresponding generalization for linear combinations. Once normalized to unity, the states constructed using $\mathfrak{Q}$, are equivalent to the symmetrized vectors for bosonic states and we have shown that commutation relations equivalent to the usual ones hold, thus being both formulations equivalent for bosons.

For fermions, there are some subtleties involved in the construction. First of all, let us recall the action of the creation operator $c_{\alpha}^{\dagger}$ : let $\zeta$ represent a collection of indexes with non null occupation number, then $\left.C_{\alpha}^{\dagger} \mid \zeta\right)=$ $\mid \alpha \zeta)$. If $\alpha$ was already in the collection $\zeta$, then $\mid \alpha \zeta$ ) is a vector with null norm. As said above, to have null norm implies that $(\psi \mid \alpha \zeta)=0$ for all $\mid \psi)$. Moreover, if a linear combination of null norm vectors were added to the vector representing the state of a system, this addition would not give place to observable results because the terms of null norm do not contribute to the mean values or to the probabilities. In order to express this situation, we define the following relation:

Definition 3.4 Two vectors $\mid \varphi$ ) and $\mid \psi$ ) are similar (and we will write $(\varphi) \cong(\psi))$ ) if the difference between them is a linear combination of null norm vectors.

With all of this, it is straightforward to demonstrate the equivalence of the anticommutation relations in $\mathbb{V}_{Q}$ and in the standard Fock-space. Thus, we can conclude that both formulations are equivalent also for fermions.

To avoid particle labeling in the expressions for observables, in Fockspace formalism they are written in terms of creation and annihilation operators. This is also the case in $\mathbb{V}_{Q}$. For example, we have shown that operators $T$ acting over a single particle states are of the form:

$T=\sum_{\alpha \beta} t_{\alpha \beta} a_{\alpha}^{\dagger} a_{\beta}=\sum_{k}(\alpha \mid k) t_{k}(k \mid \beta) a_{\alpha}^{\dagger} a_{\beta}=\sum_{k} \sum_{j}(\alpha \mid k)(k|T(1)| j)(j \mid \beta) a_{\alpha}^{\dagger} a_{\beta}$

Interaction operators act over spaces of a greater number of particles. The 
expression of an interaction operator $V$ between two particles is:

$$
V=\frac{1}{2} \sum_{\alpha} \sum_{\beta} \sum_{\gamma} \sum_{\delta} V_{\alpha \beta, \gamma \delta} a_{\alpha}^{\dagger} a_{\beta}^{\dagger} a_{\gamma} a_{\delta}=\frac{1}{4} \sum_{\alpha} \sum_{\beta} \sum_{\gamma} \sum_{\delta}(\kappa \lambda|V| \mu \nu) a_{\alpha}^{\dagger} a_{\beta}^{\dagger} a_{\gamma} a_{\delta}
$$

\section{Correlation in a two-particle state}

In this section we show an application of the use of the formalism in $\mathbb{V}_{Q}$ to illustrate how the usual results of standard QM formalism are obtained. To do so, let us consider a two spin $1 / 2$ quanta regarding only to spin degrees of freedom. Let $S_{i}=(\hbar / 2) \sigma_{i}$ be the spin operator, $\sigma_{i}$ the Pauli matrices. We

use eq. (1) to write $\sigma_{z}: \sigma_{z}=\sum_{\alpha \beta}\left(\sigma_{z}\right)_{\alpha \beta} C_{\alpha}^{\dagger} C_{\beta}=C_{+}^{\dagger} C_{+}-C_{-}^{\dagger} C_{-}$. To obtain the spin operator in an arbitrary direction $\hat{n}=(\sin \theta \cos \phi, \sin \theta \sin \phi, \cos \theta)$, we propose that $\sigma_{n}$ is of the form:

$$
\sigma_{n}=\cos \theta C_{+}^{\dagger} C_{+}+e^{-i \phi} \sin \theta C_{+}^{\dagger} C_{-}+e^{i \phi} \sin \theta C_{-}^{\dagger} C_{+}-\cos \theta C_{-}^{\dagger} C_{-}
$$

In fact, this operator rotates the basis vectors as usual. Thus, the mean value of $\sigma_{n}$ in the one particle state 'up' in direction $\hat{z}$ results:

$$
\left(+\left|\sigma_{n}\right|+\right)=\cos \theta(+\mid+)+e^{i \phi} \sin \theta(+\mid-)=\cos \theta
$$

Now we consider the a pair of indistinguishable fermions, one with spin 'up' and the other with spin 'down'. In $\mathbb{V}_{Q}$ its state is $\left.\mid+-\right)$. It has not to be confused with the standard $|+-\rangle$, which is not an antisymmetric state. We first show that in the same spatial direction, say $\hat{z}$, the spin components are in perfect anticorrelation. As usual, the correlation is evaluated as the mean value of an operator that represents the measurement of $\sigma_{z}$ for both components over the state. Differently from the standard formulation, where this operator is obtained in the labeled tensor product space, here it is obtained from eq. (2) for the fermionic case:

$$
\begin{aligned}
\sigma_{\mathbf{z z}} & =\frac{1}{2} \sum_{\alpha} \sum_{\beta} \sum_{\gamma} \sum_{\delta}\left(\sigma_{z z}\right)_{\alpha \beta, \gamma \delta} C_{\alpha}^{\dagger} C_{\beta}^{\dagger} C_{\delta} C_{\gamma} \\
& =\frac{1}{2}\left[C_{+}^{\dagger} C_{+}^{\dagger} C_{-} C_{+}-C_{+}^{\dagger} C_{-}^{\dagger} C_{+} C_{-}+C_{-}^{\dagger} C_{-}^{\dagger} C_{-} C_{-}-C_{-}^{\dagger} C_{+}^{\dagger} C_{+} C_{-}\right]
\end{aligned}
$$


When applied to a state $\mid+-$ ) it yields

$$
\begin{aligned}
\left.\sigma_{\mathbf{z z}} \mid+-\right) & \left.=\frac{1}{2}\left[C_{+}^{\dagger} C_{+}^{\dagger} C_{+} C_{+} \mid+-\right)-C_{+}^{\dagger} C_{-}^{\dagger} C_{-} C_{+} \mid+-\right) \\
& \left.\left.\left.+C_{-}^{\dagger} C_{-}^{\dagger} C_{-} C_{-} \mid+-\right)-C_{-}^{\dagger} C_{+}^{\dagger} C_{+} C_{-}\right] \mid+-\right) \\
& \left.\left.\left.=\frac{1}{2}[-\mid+-)+\mid-+\right)\right]=-\mid+-\right)
\end{aligned}
$$

Thus, the mean value results $\left(+-\left|\sigma_{\mathbf{z z}}\right|+-\right)=-(+-\mid+-)=-1$, which is the usual result.

To obtain the correlation between components in two arbitrary directions, say $\hat{z}$ and $\hat{n}$, we have to follow an analogous procedure. First we write the operator $\sigma_{z n}$ that acts over the state space of the two particles without distinguishing them:

$$
\begin{aligned}
\sigma_{\mathbf{z n}} & =\frac{1}{2}\left[\cos \theta C_{+}^{\dagger} C_{+}^{\dagger} C_{+} C_{+}+e^{-i \phi} \sin \theta C_{+}^{\dagger} C_{+}^{\dagger} C_{-} C_{+}\right. \\
& +e^{i \phi} \sin \theta C_{+}^{\dagger} C_{-}^{\dagger} C_{+} C_{+}-\cos \theta C_{+}^{\dagger} C_{-}^{\dagger} C_{-} C_{+} \\
& +\cos \theta C_{-}^{\dagger} C_{-}^{\dagger} C_{-} C_{-}-e^{-i \phi} \sin \theta C_{-}^{\dagger} C_{-}^{\dagger} C_{+} C_{-} \\
& \left.-e^{-i \phi} \sin \theta C_{-}^{\dagger} C_{+}^{\dagger} C_{-} C_{-}-\cos \theta C_{-}^{\dagger} C_{+}^{\dagger} C_{+} C_{-}\right]
\end{aligned}
$$

Applied to the state $\mid+-)$ it yields:

$$
\begin{aligned}
\left.\sigma_{\mathbf{z n}} \mid+-\right) & \left.=\frac{1}{2}\left[e^{-i \phi} \sin \theta C_{+}^{\dagger} C_{+}^{\dagger} \mid 0\right)-\cos \theta C_{+}^{\dagger} C_{-}^{\dagger} \mid 0\right) \\
& \left.\left.\left.+e^{-i \phi} \sin \theta C_{-}^{\dagger} C_{-}^{\dagger} \mid 0\right)+\cos \theta C_{-}^{\dagger} C_{+}^{\dagger} \mid 0\right)\right] \\
& \left.\left.\left.\left.=\frac{1}{2}[-\cos \theta \mid+-)+\cos \theta \mid-+\right)\right]=\frac{1}{2}[-\cos \theta \mid+-)-\cos \theta \mid+-\right)\right] \\
& =-\cos \theta \mid+-)
\end{aligned}
$$

Thus, the mean value which gives the correlation results $\left(+-\left|\sigma_{\mathbf{z n}}\right|+-\right)=$ $-\cos \theta$, as it must be. It is important to remark that the state $\mid+-$ ) takes into account indistinguishability and antisymmetry without 'tricks', just because it is constructed in $\mathbb{V}_{Q}$.

\section{Conclusions}

We have argued that it is possible to construct a quantum mechanical formalism for indistinguishable particles making use of quasi-set theory $\mathfrak{Q}$ to 
build a vector space $\mathbb{V}_{Q}$ that resembles the Fock-space but without labeling quanta in any step. In $\mathbb{V}_{Q}$, states refer only to occupation numbers and permutations of quanta are unobservable. In this paper, we have exemplified the use of the new formalism to evaluate the correlations between the spin components of a two-fermions system, explicitly showing that it is not necessary to first impose labels to the particles and then masking the individuation by a symmetrization postulate to obtain the usual results.

\section{References}

[1] Aerts, D. and Daubechies, I. "Structure-preserving maps of a quantum mechanical propositional system", Helvetica Physica Acta 51, pp. 637660, 1978.

[2] Domenech, G., Holik, F. and Krause, D., "Quasi-spaces and the foundations of quantum mechanics", Foundations of Physics, in press (2008).

[3] Domenech, G., Holik, F. and Kniznik, L., preprint (2008).

[4] Enderton, H. B., Elements of set theory (Academic Press, 1977).

[5] French, S. y Krause, D., Identity in physics: a historical, philosophical, and formal analysis (Oxford University Press, 2006).

[6] Krause, D., "Logical aspects of quantum (non-)individuality", preprint.

[7] Krause, D., Sant'Anna, A. y Sartorelli, A., "A critical study on the concept of identity in Zermelo-Fraenkel like axioms and its relationship with quantum statistics", Logique and Analyse, 189-192, 231-260 (2005).

[8] Robertson, B., "Introduction to field operators in quantum mechanics", American Journal of Physics 41, 678 (1973). 